\title{
Análisis del impacto de la propiedad intelectual en el desarrollo económico en países de América, Asia y Europa
}

\section{Analysis of the impact of intellectual property on economic development in the countries of America, Asia and Europe}

\author{
S. Gayosso-Mexia ${ }^{a}$, C. Muñoz-Ibáñez, ${ }^{b}$ A. Carrizal-Alonsoc, F. Benitez-Leal ${ }^{d}$
}

\begin{abstract}
:
At the beginning of the new millennium, economic development in favor of improving the standard of living of the world's inhabitants continues to be a complex challenge, which has led the business environment to reinvent itself, through the generation of new knowledge, through Research and Development (R\&D) activities, which have resulted in innovations that allow impact within regional, national and international markets. Within this context, technological innovation and creative activity are fundamental sources of progress and growth, and, therefore, intellectual property has become increasingly important, in that its protection has become a tool for promote the creation of knowledge and its respective international transfer. Under the previous premise, the industrial property protection figures such as trademarks, patents, utility models and industrial designs take on a substantial relevance, since they are mechanisms that ensure the protection of innovations and their economic exploitation, resulting in the development and the economic growth of the countries that in turn are favored within the economic balance. This research focuses on the development of a linear forecasting model that helps assess the direct impact that industrial protection figures have on the growth and economic development of countries such as Germany, Argentina, Brazil, China, Spain, the United States, America, France, India, Japan, adding to Mexico to compare it with international activity.
\end{abstract}

Keywords:

Intellectual Property, Development, Growth.

\section{Resumen:}

A inicios del nuevo milenio el desarrollo económico en favor de mejorar el nivel de vida de los habitantes del mundo continúa siendo un desafío complejo, por lo que ha llevado al entorno empresarial a reinventarse, a través de la generación de nuevos conocimientos, por medio de actividades de Investigación y Desarrollo (I+D), que han tenido como resultado innovaciones que permiten impactar dentro de los mercados regionales, nacionales e internacionales. Dentro de este contexto, la innovación tecnológica y la actividad creadora constituyen fuentes fundamentales de progreso y crecimiento, y, por lo tanto, la propiedad intelectual ha venido adquiriendo cada vez más importancia, es por cuanto que su protección se ha convertido en una herramienta para promover la creación del conocimiento y su respectiva transferencia internacional. Bajo la premisa anterior las figuras de protección de la propiedad industrial como son marcas, patentes, modelos de utilidad y diseños industriales toman una relevancia sustancial, ya que son mecanismos que aseguran la protección de las innovaciones y su explotación económica, dando como resultado el desarrollo y el crecimiento económico de los países que a su vez se ven favorecidos dentro de la balanza económica. Esta investigación se centra en el desarrollo de un modelo de pronóstico lineal que ayude a evaluar el impacto directo que tienen las figuras de la protección industrial con el crecimiento y desarrollo económico de países como Alemania, argentina, Brasil, China, España, Estados Unidos de América, Francia, India, Japón, agregando a México para compararlo con la actividad internacional.

Palabras Clave:

Propiedad Intelectual, Desarrollo Económico, Crecimiento.

a Autor de Correspondencia, Universidad Autónoma del Estado de Hidalgo, Escuela Superior de Tizayuca, https://orcid.org/0000-00025428-872X, Email: sonia_gayosso8990@uaeh.edu.mx

b, c. d Universidad Autónoma del Estado de Hidalgo, Escuela Superior de Tizayuca, https://orcid.org/0000-0002-3088-2599, https://orcid.org/0000-0002-2001-1001, https://orcid.org/0000-0003-1111-5340, Email: cristopher_munoz@, aide_carrizal8989@, 


\section{Introducción}

En el desarrollo económico del mundo influyen diversos factores sociales, tecnológicos y mercadológicos; estos factores intervienen en la creación de normas que impulsan y regulan la economía, aseguran la compatibilidad y competitividad dentro de la comercialización, lo que implica la importación y exportación de productos.

Dentro de la dinámica comercial antes descrita, existen cuatro tipos de propiedad intelectual (PI), las patentes, los modelos de utilidad, los diseños industriales (modelos o dibujos industriales) y las marcas; a nivel mundial se procesan los datos de las oficinas nacionales y regionales de Propiedad Intelectual, del Banco Mundial y de la Organización Mundial de la Propiedad Intelectual (OMPI) para generar indicadores mundiales que hacen referencia a la PI en todo el mundo.

Con lo anterior es posible comprender que la propiedad industrial actúa positivamente en el aumento del Producto Interno Bruto (PIB), que genera determinado país, el cual vinculado con la paridad de poder adquisitivo funciona como un indicador que permite conocer el nivel de vida de una nación.

\section{Panorama internacional del efecto de la propiedad industrial en el desarrollo económico}

Las innovaciones tecnológicas y la apertura del comercio han modificado profundamente el panorama de la producción mundial. Convertir materias primas en piezas y componentes, ensamblar los productos finales y hacerlos llegar al consumidor final movilizan cadenas de suministro que se extienden por un número creciente de economías de todo el mundo [1].

Actualmente los países desarrollados económicamente están orientados en áreas de investigación y desarrollo, las cuales están enfocadas en crear invenciones y protegerlas, por lo cual cuentan con diversas organizaciones locales e internacionales, que permiten compartir el conocimiento y crear sistemas económicos sostenibles y viables para su región [2]. Hoy en día el panorama económico es bastante optimista con respecto al crecimiento de las innovaciones y el aumento en el gasto de $\mathrm{I}+\mathrm{D}$, que, de acuerdo con investigaciones realizadas, tan solo del año 1996 al 2006 se duplico con creces el gasto [3].

Continuando con el autor anterior, es igual de importante mencionar que la iniciativa privada tiene un papel preponderante en el crecimiento económico, pues en el año 2016 registra a nivel mundial un aumento de gasto empresarial de I+D del $4.2 \%$.

De acuerdo a los Indicadores Mundiales de Propiedad Intelectual del año 2014 al 2015 hubo un crecimiento pronunciado de la demanda de derechos de Propiedad Intelectual (P.I.); patentes aumenta el $7.8 \%$ teniendo un total de 2,9 millones, modelos de utilidad 1,2 millones lo que se traduce en un aumento del $27 \%$, marcas tiene un incremento porcentual del 13.7 que en cifras son 8,4 millones, y finalmente, los diseños industriales tuvieron el incremente más bajo en comparación con los anteriores del $0.6 \%$, mismo porcentaje que refleja 1,1 millones [4].

En el año 2017 se dan a conocer las estadísticas del crecimiento solicitudes de concesión de derecho de PI y derechos de PI vigentes a nivel mundial, dichos Indicadores Mundiales de la Propiedad Intelectual se presentan de los años 2015-2016; las patentes y los diseños industriales presentan un crecimiento del $8.3 \%$, que con respecto al año 2014 se manifiesta un evidente aumento en el número de solicitudes y derechos, los modelos de utilidad tienen 1,6 millones de solicitudes con un crecimiento del 28.9 $\%$, finalmente las marcas registran un total de 9,8 millones con un porcentaje de aumento del 13.5.

Según los resultados obtenidos por la Organización Mundial de Propiedad Intelectual [5], se ha comprobado que el servicio de propiedad industrial más utilizado son los registros de marca, esto debido al valor agregado que le brinda al producto, que simboliza la pertenencia a la persona moral o física que creo la invención [6].

Por otro lado, en cada continente el uso de la propiedad industrial se administra conforme a las normas y criterios establecidos por los participantes, contemplando sus características particulares y condiciones que benefician al crecimiento y desarrollo económico [7].

A continuación, se presentan datos estadísticos de algunos países de América, Asía y Europa, considerando que los países seleccionados 
representan una muestra de los datos presentados en los Indicadores Mundiales de Propiedad Intelectual de los años 2014-2016.

\subsection{América}

\section{Argentina}

Según la OMPI 2016, todos los países de Latinoamérica apenas registran 1,200 patentes, de las cuales 26 son Argentina, a quien su legislación le permite crear todos los tipos de propiedad industrial, ya que tiene legislaciones más consolidadas y la gestión colectiva a través de sociedades de derechos autor y conexos, está en fases maduras de implementación.

En el caso de Argentina, la propiedad intelectual juega a favor de las industrias creativas más sólidas y generadoras de empleo, como la editorial [8].

\section{Brasil}

Es en la década de los 60 s algunos de los países latinoamericanos iniciaron modificaciones en materia de legislación en materia de propiedad intelectual; Brasil, México y algunos países del grupo andino son ejemplo de ello, ya que buscaron elevar la competitividad y por ende fortalecer la propiedad intelectual. Los países de América Latina han trabajado en la reformulación de los sistemas de propiedad intelectual, esto respecto a los avances tecnológicos que cada uno de los países genera, permitiendo la evolución de la protección de la propiedad intelectual a través de sistemas más sofisticados [9]. Actualmente Brasil ha tenido un crecimiento significativo en su participación en la propiedad industrial, pues actualmente se ha coronado como el principal proponente en América Latina gracias a su incidencia en este rubro, ya que cuenta con 660 patentes hasta 2016 [10].

Lo anterior atestigua que la segunda economía del mundo hace suya sin reservas la P.I., convencida de que la innovación es la fuerza motriz del progreso económico.

\section{Estados Unidos de Norteamérica}

Este país es el proponente más importante del continente al solicitar y otorgar gran cantidad de patentes, marcas y diseños industriales, los modelos de utilidad no entran en su reglamento, por lo que solo permite los modelos de utilidad que sean extranjeros y no desarrollados en el país [8].
Los ingresos impulsados por el valor de la P.I. en la economía estadounidense representan el $38 \%$ del PIB total de la nación; lo que indica que casi cuatro de cada diez dólares de su economía se relacionan con las industrias intensivas en I.P. La exportación de mercancías, la generación de empleos y la prima salarial también se ven favorecidas por la inversión en innovación y desarrollo [7].

\section{México}

Actualmente se encuentra con una legislación que a pesar de que está a favor del desarrollo de la propiedad industrial, el principal obstáculo es la falta de difusión para acrecentar el número de solicitudes y de otorgamientos de patentes [11].

Indudablemente la poca regulación en la propiedad industrial ha puesto a México en una serie de altibajos económicos que en vez de potenciar la estabilidad para el desarrollo económico solo ha logrado la inestabilidad de este sistema [12] [13].

A principios del 2018 la cantidad de registro de patentes se incrementa a 17 mil 184 sin embargo de estas solo el ocho por ciento corresponden a inventores mexicanos en contraposición al $92 \%$ de extranjeros lo que sin duda alguna impacta en el sector económico [14].

\subsection{Asia}

\section{China}

En los treinta años transcurridos desde que China pasó a ser miembro de la Organización Mundial de la Propiedad Intelectual (OMPI), el país ha registrado notables avances en el campo de la propiedad intelectual (P.I.) a tal punto que su oficina de patentes figura entre las cinco primeras del mundo y la oficina de marcas es la más grande del planeta [15].

Desde su instauración en 1985, el sistema chino de patentes ha evolucionado de forma considerable, habiéndose batido nuevas marcas y mejorando notablemente la capacidad del país en el campo de la innovación. En el primer decenio del siglo XXI, las solicitudes de patente registraron un aumento anual promedio del $22,3 \%$, a tal punto que, solamente de enero a octubre de 2010, fueron presentadas 295.275 patentes de invención, es decir, el $25 \%$ del mismo período de 2009, y de ellas, casi tres cuartos del total 
(72,5\%, o sea, 214.079 solicitudes) correspondían a solicitantes del país [16].

\section{India}

En cuanto a los múltiples factores que intervienen en el desarrollo económico India también ha incursionado en el mundo que engloba la protección industrial, a diferencia de otras legislaciones con respecto a la propiedad industrial, India no irrumpe en los modelos de utilidad por lo que internamente no se encargan de elaborarlos y solo se otorgan a los extranjeros [10]. A pesar de lo anterior, el desarrollo ha ido en ascenso a lo largo de los años en este país, permitiendo tener posibilidades de crecer y convertirse en una potencia mundial reconocida e incidir en los mercados.

\begin{abstract}
Japón
A pesar del poco espacio territorial que tiene este país, no ha dejado de crecer notablemente en los últimos años, uno de los elementos esenciales y vitales para el desarrollo económico de Japón, fue la Política Industrial creada para la incentivación, invertir, proteger y fomentar la propiedad industrial, esto ha permitido el crecimiento constante en el ámbito industrial, accediendo así a un desarrollo económico estable, colocándolo como un país con productos de calidad y capaces de exportarse, incidiendo en muchos mercados creando así un aumento constante en su PIB [17].
\end{abstract}

\subsection{Europa}

En Europa, la propiedad industrial ha tomado un papel importante para los países que quieran incursionar entre las grandes potencias mundiales, debido a la importancia de las aportaciones económicas e intelectuales que presenta [2].

\section{Alemania}

En el 2017 Alemania fu considerada la economía más grande de Europa y una de las principales potencias mundiales, el factor más importante para su desarrollo como país se ha concentrado esencialmente en la protección industrial, por lo que se convirtió en un foco central en los decretos para promoción y protección recíproca de inversiones. Cabe resaltar que esto ha impactado en el desarrollo económico del país, por lo que el Secretario de Economía reafirma que la paridad de poder adquisitivo permitirá mantener el nivel de vida del territorio alemán de forma sostenible [18].

\section{España}

La finalidad de realizar proyecto de $\mathrm{I}+\mathrm{D}+\mathrm{i}$ por parte de una empresa es la obtención de una serie de resultados que le permitan ganar competitividad a través de nuevos o mejorados productos, servicios o procesos. Pero la ventaja competitiva existe siempre y cuando los resultados y el conocimiento relacionado no sean conocidos por los competidores o, en su caso, aunque sean conocidos, que éstos estén limitados en su utilización o aplicación [19].

Derivado de lo anterior la Comisión Europea detalla que en España las industrias que dependen para su desarrollo de los derechos de autor aportan más del $3 \%$ del total del empleo (780.000 profesionales) y suponen un 3,6 \% del Producto Interior Bruto, un porcentaje superior al generado por la agricultura, ganadería y pesca (2,5\%), la industria química $(1,1 \%)$ o las telecomunicaciones (1,8 \%) [19].

\section{Francia}

En el entorno francés la propiedad industrial es un elemento que se ha convertido en el principal componente para el desarrollo económico, Francia es considerada la quinta potencia económica mundial y la segunda fuerza económica de Europa. Así, es posible resaltar parte de la estabilidad que han tenido como país, aunque para el año 2015 tuvo un crecimiento porcentual del 1.2 de PIB [20]

\section{Investigaciones relacionadas}

El sistema de Propiedad Industrial se materializa en la concesión de derechos exclusivos a sus titulares, derechos que les otorgan privilegios, pero también deberes, también hace referencia a derechos que protegen a los creadores de invenciones para que puedan desarrollar su actividad en el mercado frente a los competidores y cuenten con una protección suficiente frente a terceros.

Existe diversidad en cuanto a los modelos que se utilizan en la propiedad industrial como el principal factor para impulsar al desarrollo económico, en esta investigación se enfatizó en modelos cuantitativos los cuales se describen a continuación:

\subsection{Modelos Cuantitativos}




\subsubsection{El negocio de la Propiedad Industrial: Modelo del IPB}

Este modelo se orienta en la adquisición de derechos del autor y su aprovechamiento de la invención por cierta periodicidad, además de enfocar la buena inversión en este y su re-contribución futura [21] [22].

Las ventajas que presenta este modelo es la obtención monetaria que crea por cada producto, modelo de utilidad, diseño industrial, patente y registro de marca el cual da un valor agregado a una invención.

Los datos referenciales son los mismos obtenidos por la OMPI ya que son en tiempo real y verídicos, de tal manera que llegan directamente de los diversos institutos de los diferentes países miembros de esta organización, lo que les permite dar a conocer la paridad de poder adquisitivo del PIB permitiendo crear una visión del nivel de vida de un país.

Al generalizar estos datos permite confirmar de manera fácil y sencilla como es importante la inversión en la propiedad industrial en diversos mercados del mundo.

\subsubsection{Modelo Propiedad industrial y desarrollo económico}

Este modelo se basa en una comparación de la importancia de la propiedad industrial para el desarrollo económico [23]; algo relevante de este modelo es la propuesta de la diferencia de servicios y comercios que dan un valor agregado no solo a la empresa o inventor, sino también a la invención para su colocación en el mercado lo que posteriormente dejara ganancias para el lugar de origen y el creador de esta.

Dentro de las características que se evalúan en esta propuesta se encuentran la económica, que es donde se involucra el Producto Interno Bruto, a pesar de ser generalizado destaca que la mayor parte de este generada por el comercio interior y exterior, lo que probablemente te hace pensar en la presencia de un producto o servicio en un mercado nacional e internacional.

\section{Propuesta del modelo}

Para el desarrollo del modelo, se seguirá el siguiente procedimiento:

1. Se definirán los principales elementos de la propiedad industrial, así como su impacto dentro del Producto Interno Bruto originado de patentes en paridad de poder adquisitivo en dólares.

2. Establecimiento de los principales países que serán considerados para la evaluación.

3. Consulta de la base de datos de la OMPI con respecto a las figuras de la propiedad industrial.

4. Desarrollo del modelo de regresión y estimación del error del modelo y análisis de los datos.

Cada uno de los puntos se desarrolla en las secciones siguientes.

\subsection{Principales elementos de la propiedad industrial y su impacto dentro del Producto Interno Bruto originado de patentes en paridad al poder adquisitivo en dólares.}

Dentro de esta sección se realizará primeramente la definición de las principales figuras de la propiedad industrial que serán analizadas y el impacto que pueden tener dentro de Producto Interno Bruto (PIB). En la propiedad industrial existen Marcas, patentes, modelos de utilidad (conocido también como pequeña patente) y diseños industriales, estos se definen como sigue [24]:

- Marcas

Es importante señalar que la marca es lo que reconoce el producto y con lo que la gente te reconoce.

- Patentes

Son los derechos que se le dan al creador de una invención a cambio de la divulgación de la misma.

- Diseños Industriales

Es el aspecto ornamental o estético del artículo de manera tridimensional y bidimensional.

- Modelos de Utilidad

Es una invención que consiste en dar aun objeto una configuración, estructura o constitución de lo que resulte alguna ventaja prácticamente.

Una vez establecidos estos conceptos es posible definir al PIB originado de patentes en paridad de poder adquisitivo en dólares como aquel que mide el 
valor de los bienes y servicios de un país, originados de la actividad inventiva que resulta en el desarrollo de alguna de las formas de propiedad industrial descritas anteriormente, tomando en consideración la variación de los precios en dólares [25].

Definidos estos conceptos, es posible ahora determinar las regiones en las cuales se llevará a cabo el estudio del impacto de la propiedad industrial en el desarrollo económico.

\subsection{Principales países que serán considerados para la evaluación.}

En la actualidad se perfilan tres bloques económicos principales, el primero es aquel que forman Estados Unidos, México y Canadá, el cual tiene la perspectiva de incorporar otros países de América Latina, el bloque europeo que está liderado por Alemania e integrado por países de la Comunidad Europea y el asiático, con Japón y China como máximos representados.

Por lo anterior el presente modelo toma como referencia los siguientes países contenidos en estos bloques económicos.

\section{- América}

- Argentina

- Brasil

- Estados Unidos

- México

- Asia

- China

- India

- Japón

- Europa
- Alemania
- España
- Francia

\subsection{Base de datos conforme la WIPO para la propiedad industrial.}

El conjunto de datos de la presente propuesta se presenta en la Tabla 1:

\begin{tabular}{|c|c|c|c|c|c|}
\hline & & $\begin{array}{l}\text { Patentes } \\
\text { Totales }\end{array}$ & & & $\begin{array}{c}\text { patentes } \\
\text { en dólares } \\
\text { paridad de } \\
\text { poder } \\
\text { adquisitivo }\end{array}$ \\
\hline \multirow[t]{4}{*}{ Japón } & 2013 & $529,846.00$ & $10,901.00$ & $366,689.00$ & $473,441.00$ \\
\hline & 2014 & $526,001.00$ & $10,404.00$ & $415,927.00$ & $475,030.00$ \\
\hline & 2015 & $517,834.00$ & $9,290.00$ & $525,917.00$ & $480,823.00$ \\
\hline & 2016 & $523,604.00$ & $8,280.00$ & $622,072.00$ & $485,624.00$ \\
\hline \multirow[t]{4}{*}{ China } & 2013 & $854,296.00$ & $893,749.00$ & $1,940,908.00$ & $1,622,239.00$ \\
\hline & 2014 & $964,859.00$ & $869,956.00$ & $2,358,580.00$ & $1,740,624.00$ \\
\hline & 2015 & $1,144,227.00$ & $1,129,160.00$ & $3,059,652.00$ & $1,860,731.00$ \\
\hline & 2016 & $1,380,961.00$ & $1,477,686.00$ & $4,199,467.00$ & $1,985,400.00$ \\
\hline \multirow[t]{4}{*}{ Brasil } & 2013 & $32,773.00$ & $3,065.00$ & $155,897.00$ & $313,896.00$ \\
\hline & 2014 & $32,395.00$ & $2,772.00$ & $150,677.00$ & $302,064.00$ \\
\hline & 2015 & $32,148.00$ & $2,749.00$ & $152,225.00$ & $291,206.00$ \\
\hline & 2016 & $30,033.00$ & $2,980.00$ & $155,168.00$ & $291,206.00$ \\
\hline \multirow[t]{4}{*}{ Argentina } & 2013 & $5,051.00$ & 198.00 & $48,680.00$ & $82,877.00$ \\
\hline & 2014 & $4,964.00$ & 179.00 & $51,903.00$ & $80,795.00$ \\
\hline & 2015 & $4,468.00$ & 163.00 & $61,006.00$ & $82,934.00$ \\
\hline & 2016 & $3,848.00$ & 224.00 & $63,751.00$ & $81,028.00$ \\
\hline \multirow[t]{4}{*}{ México } & 2013 & $16,373.00$ & 740.00 & $100,934.00$ & $199,928.00$ \\
\hline & 2014 & $17,076.00$ & 721.00 & $108,718.00$ & $204,457.00$ \\
\hline & 2015 & $19,216.00$ & 686.00 & $121,244.00$ & $209,833.00$ \\
\hline & 2016 & $18,417.00$ & 718.00 & $126,225.00$ & $214,665.00$ \\
\hline \multirow[t]{4}{*}{ Alemania } & 2013 & $200,307.00$ & $16,475.00$ & $2,193,102.00$ & $346,086.00$ \\
\hline & 2014 & $102,205.00$ & $15,919.00$ & $2,103,796.00$ & $351,608.00$ \\
\hline & 2015 & $194,932.00$ & $15,282.00$ & $2,214,047.00$ & $357,658.00$ \\
\hline & 2016 & $196,492.00$ & $15,035.00$ & $2,300,068.00$ & $364,336.00$ \\
\hline
\end{tabular}

Tabla 1. Datos de los principales países con respecto a la propiedad industrial (Fuente: Recopilación de datos de la OMPI)

Desarrollo del modelo de regresión y estimación del error del modelo y análisis de los datos.

Una vez realizada la base datos es posible hacer la estimación del impacto que tiene las diferentes figuras de la propiedad industrial en el PIB que se registra, para esta relación será utilizado el coeficiente de regresión lineal de Pearson como se muestra en la ecuación 1.

$$
\frac{n \sum_{i=1}^{n} x_{i} y_{i}-\sum_{i=1}^{n} x_{i} \sum_{i=1}^{n} y_{i}}{\sqrt{\left[n \sum_{i=1}^{n} x_{i}^{2}-\left(\sum_{i=1}^{n} x_{i}\right)^{2}\right]\left[n \sum_{i=1}^{n} y_{i}^{2}-\left(\sum_{i=1}^{n} y_{i}\right)^{2}\right]}}
$$

Al aplicar el coeficiente de regresión lineal, se obtiene que aquel que tiene un mayor impacto para el crecimiento del PIB son la solicitudes de patente con 0.95 , en segunda instancia los modelos de utilidad con 0.96 y por último los registros de marca con el 0.78 , lo cual establece la correlación lineal que existe entre estos factores y el PIB, con lo cual estos tres factores serán considerados para construir el modelo de pronóstico el cual se realizara utilizando una regresión lineal que satisface la ecuación 2.

$$
y_{i}=\beta_{0}+\beta_{1} x_{i}+\epsilon_{i}
$$


Una vez estimado los diferentes coeficientes de $\beta_{0} y \beta_{1}$ se obtienen los valores para cada modelo de regresión lineal que se muestran en la Tabla 2.

\begin{tabular}{|c|r|r|r|}
\hline Coeficiente & \multicolumn{1}{|c|}{$\begin{array}{c}\text { Sol. Pat / } \\
\text { PIB }\end{array}$} & $\begin{array}{c}\text { Marca / } \\
\text { PIB }\end{array}$ & Modelo / PIB \\
\hline$\beta_{0}$ & 223510.77 & 301744.35 & 518379.8633 \\
\hline$\beta_{1}$ & 1.42 & 0.38 & 0.99 \\
\hline
\end{tabular}

Tabla 2. Estimación de los coeficientes de la regresión lineal (Fuente: Elaboración propia)

Una vez aplicados los estimadores se obtienen los resultados que se muestran en la Tabla 3 , donde se pueden apreciar los pronósticos que presenta para determinar el crecimiento del producto interno bruto utilizando cada uno de los modelos desarrollados en la Tabla 2, esto permitirá hacer una evaluación de manera individual por cada factor y su impacto dentro del crecimiento económico a través del producto interno bruto. Por otro lado, es importante mencionar que a pesar de que los modelos de utilidad tienen un impacto significativo dentro del PIB, empezando su crecimiento a partir de los 518,379.86, son las solicitudes de patente las que presentan el mayor gradiente de crecimiento, lo que marca la importancia de esta figura de la protección de la propiedad industrial.

\begin{tabular}{|c|c|c|c|c|}
\hline País & Año & $\begin{array}{c}\text { Pronóstico } \\
\text { (PIB / } \\
\text { Solicitudes } \\
\text { de } \\
\text { Patentes) }\end{array}$ & $\begin{array}{c}\text { Pronóstico } \\
\text { (Marca / } \\
\text { PIB) }\end{array}$ & $\begin{array}{l}\text { Pronóstico } \\
\text { (Modelos } \\
\text { de Utilidad } \\
\text { /PIB) }\end{array}$ \\
\hline \multirow[t]{4}{*}{ Japón } & 2013 & 973,903 & 441,886 & 529,122 \\
\hline & 2014 & 968,457 & 460,704 & 528,632 \\
\hline & 2015 & 956,891 & 502,740 & 527,534 \\
\hline & 2016 & 965,062 & 539,489 & 526,539 \\
\hline \multirow[t]{4}{*}{ China } & 2013 & $1,433,403$ & $1,043,524$ & $1,399,096$ \\
\hline & 2014 & $1,589,988$ & $1,203,150$ & $1,375,650$ \\
\hline & 2015 & $1,844,017$ & $1,471,087$ & $1,631,075$ \\
\hline & 2016 & $2,179,290$ & $1,906,703$ & $1,974,519$ \\
\hline \multirow[t]{4}{*}{$\begin{array}{l}\text { Estados } \\
\text { Unidos }\end{array}$} & 2013 & $1,335,183$ & 868,732 & $N / A$ \\
\hline & 2014 & $1,361,077$ & 884,587 & $\mathrm{~N} / \mathrm{A}$ \\
\hline & 2015 & $1,401,454$ & 942,892 & $N / A$ \\
\hline & 2016 & $1,401,892$ & 917,558 & $\mathrm{~N} / \mathrm{A}$ \\
\hline \multirow[t]{4}{*}{ Brasil } & 2013 & 269,925 & 361,325 & 521,400 \\
\hline & 2014 & 269,390 & 359,330 & 521,111 \\
\hline & 2015 & 269,040 & 359,922 & 521,089 \\
\hline & 2016 & 266,045 & 361,047 & 521,316 \\
\hline \multirow[t]{3}{*}{ Argentina } & 2013 & 230,664 & 320,349 & 518,575 \\
\hline & 2014 & 230,541 & 321,581 & 518,556 \\
\hline & 2015 & 229,839 & 325,060 & 518,540 \\
\hline
\end{tabular}

\begin{tabular}{ccccc}
\multirow{4}{*}{ México } & 2016 & 228,960 & 326,109 & 518,601 \\
& 2013 & 246,699 & 340,319 & 519,109 \\
& 2014 & 247,695 & 343,294 & 519,090 \\
& 2015 & 250,725 & 348,082 & 519,056 \\
\multirow{4}{*}{ Alemania } & 2016 & 249,594 & 349,985 & 519,087 \\
& 2013 & 507,195 & $1,139,908$ & 534,615 \\
& 2014 & 368,258 & $1,105,776$ & 534,067 \\
& 2015 & 499,582 & $1,147,912$ & 533,439 \\
& 2016 & 501,792 & $1,180,788$ & 533,196 \\
& 2013 & 298,954 & 380,087 & N/A \\
& 2014 & 298,939 & 392,415 & N/A \\
& 2015 & 304,334 & 410,034 & N/A \\
& 2016 & 305,232 & $1,427,647$ & N/A
\end{tabular}

Tabla 3. Pronósticos de los modelos lineales (Fuente: Elaboración propia)

Nota: Estados unidos e India en su legislatura de protección de propiedad intelectual no reconocen los modelos de utilidad por lo cual se coloca no aplica en el modelo (N/A)

\section{Conclusiones}

Los modelos desarrollados permiten visualizar la importancia que existe de las diversas figuras de la propiedad industrial dentro del desarrollo económico de los países, estos elementos se encuentran intrínsecos dentro del crecimiento de los países e influyen directamente en el bienestar de las naciones.

Por otro lado, en el presente trabajo fue posible identificar de manera cuantitativa que existe un efecto importante de la propiedad industrial sobre el crecimiento del PIB, y se construyeron tres modelos lineales para su estimación, de estos modelos, los modelos de utilidad muestran un impacto directo dentro del crecimiento del PIB, posteriormente las patentes son los elementos que permiten el mayor gradiente de crecimiento y por último las marcas muestran un crecimiento pequeño pero constante en el PIB.

Por último, es conveniente mencionar que, aunque las actividades de investigación y desarrollo realizadas por las empresas y las naciones son procesos costosos y que tienen un gran grado de incertidumbre, aseguran un bienestar al largo plazo que se refleja en los datos del modelo presentado, por cual las políticas públicas orientadas a investigación tienen que ser establecidas y evaluadas al mediano y largo plazo para determinar de manera efectiva su impacto.

\section{Referencias}

D. J. Hemel and L. L. Ouellette, "Innovation Policy Pluralism," Yale L.J., vol. 128, no. 3, pp. 544-614, 2019. 
[2] Organización Mundial de la Propiedad Intelectual, "Índice Mundial de Innovación 2017: Suiza, Suecia, los Países Bajos, los EE.UU. y el Reino Unido encabezan el ranking anual," 2017. .

[3] C. Jewell, "Índice Mundial de Innovación 2018: 'La innovación es energía,"” 2018.

[4] Organización Mundial de la Propiedad Intelectual, Datos y cifras de la OMPI sobre P.I. 2016. 2016.

[5] Organización Mundial de la Propiedad Intelectual, "Estadísticas de propiedad intelectual," 2016. .

[6] Organización Mundial de la Propiedad Intelectual, "El negocio de la propiedad intelectual," 2016. [Online]. Available: https://www.wipo.int/ipadvantage/es/details.jsp?id=2 615.

[7] J. Antonipillai and M. K. Lee, "Intellectual Property and the U.S. Economy: 2016 Update," USPTO Rep., 2016.

[8] E. F. Fernando Henrique Cardoso, Dependencia y desarrollo en América Latina: ensayo de interpretación sociológica, 30th ed. México, 2002.

[9] A. González Merino, "Propiedad intelectual, diversidad biológica y desarrollo sustentable," 1999.

[10] OMPI- Organización Mundial de la Propiedad Intelectual, Capital intangible en las cadenas globales de valor. 2017.

[11] X. Ginebra, "LA PROPIEDAD INDUSTRIAL Y LA COMPETENCIA ECONÓMICA EN MÉXICO," pp. 117-171, 2007.

[12] M. Casalet, "Descentralización y desarrollo económico local: una visión general del caso de México," Com. Económica para América Lat. y el Caribe, p. 49, 2000.

[13] A. Guzmán, J. Ludlow, H. Gómez, A. Guzmán, J. Ludlow, and H. Gómez, "Brechas tecnológicas y de innovación entre países industrializados y países en desarrollo en la industria farmacéutica," Investig. económica, vol. 63, no. 248, pp. 95-145, 2004.

[14] Eduardo Vázquez Reyes, "Actividad intelectual, base para el crecimiento económico," 2018. .

[15] Fact Sheets on the European Union, "Intellectual, industrial and commercial property," no. October 2008, pp. 1-7, 2018.

[16] Organización Mundial de la Propiedad Intelectual, "Cifras récord en el número de solicitudes internacionales de patente gracias a China; Crece la demanda de protección de las marcas y dibujos o modelos industriales," 2018. [Online]. Available: https://www.wipo.int/pressroom/es/articles/2018/arti cle_0002.html. [Accessed: 28-Feb-2019].

[17] E. Rodríguez, "Rodriguez Asien, E.: “,” vol. 7, p. 15, 2015.

[18] Portal Santader Trade, "ALEMANIA: POLÍTICA Y ECONOMÍA," 2018.

[19] Oficina Española de Patentes y Marcas, ¿¿Qué es la Propiedad Industrial?," 2019.

[20] Oficina Económica y Comercial de España en París, "INFORME ECONÓMICO Y COMERCIAL," pp. 14-32, 2016.

[21] J. P. Ogier, "Propiedad intelectual, gestión financiera y desarrollo económico," Organización Mundial de la Propiedad Intelectual, 2016.

[22] Organización Mundial de la Propiedad Intelectual, "Sobre la Clasificación Internacional de Patentes," 2019. .
[23] H. Chang, "Entendiendo la relación entre las instituciones y el desarrollo económico - Algunos aspectos teóricos claves (*)," Leonardo, vol. 8, 2005.

[24] A. Gil Lázaro and M. J. Fernández Vicente, "Los discursos sobre la emigración española en perspectiva comparada. Principios del siglo XX Principios del siglo XXI," p. 39, 2015.

[25] R. Félix, "El valor de la propiedad intelectual en los países en desarrollo Interciencia," pp. 105-110, 2003. 